\title{
Imaging Formation Algorithm of the Ground and Space-Borne Hybrid BiSAR Based on Parameters Estimation from Direct Signal
}

\author{
Qingjun Zhang, ${ }^{1}$ Cheng $\mathrm{Hu}^{2}{ }^{2}$ Lixin $\mathrm{Wu},{ }^{2}$ Tao Zeng, ${ }^{2}$ and Teng Long ${ }^{2}$ \\ ${ }^{1}$ China Academy of Space Technology (CAST), Beijing 100094, China \\ ${ }^{2}$ Radar Research Lab, Beijing Institute of Technology, Beijing 100081, China \\ Correspondence should be addressed to Cheng Hu; hucheng.bit@gmail.com
}

Received 30 October 2013; Accepted 6 January 2014; Published 20 February 2014

Academic Editor: Jui-Sheng Lin

Copyright ( 2014 Qingjun Zhang et al. This is an open access article distributed under the Creative Commons Attribution License, which permits unrestricted use, distribution, and reproduction in any medium, provided the original work is properly cited.

This paper proposes a novel image formation algorithm for the bistatic synthetic aperture radar (BiSAR) with the configuration of a noncooperative transmitter and a stationary receiver in which the traditional imaging algorithm failed because the necessary imaging parameters cannot be estimated from the limited information from the noncooperative data provider. In the new algorithm, the essential parameters for imaging, such as squint angle, Doppler centroid, and Doppler chirp-rate, will be estimated by full exploration of the recorded direct signal (direct signal is the echo from satellite to stationary receiver directly) from the transmitter. The Doppler chirp-rate is retrieved by modeling the peak phase of direct signal as a quadratic polynomial. The Doppler centroid frequency and the squint angle can be derived from the image contrast optimization. Then the range focusing, the range cell migration correction (RCMC), and the azimuth focusing are implemented by secondary range compression (SRC) and the range cell migration, respectively. At last, the proposed algorithm is validated by imaging of the BiSAR experiment configured with china YAOGAN 10 SAR as the transmitter and the receiver platform located on a building at a height of $109 \mathrm{~m}$ in Jiangsu province. The experiment image with geometric correction shows good accordance with local Google images.

\section{Introduction}

Recently, bistatic synthetic aperture radar has been a hot topic because of its advantage of flexible configuration, abundant information, anti-interception, anti-interference, and so forth. According to the platforms of BiSAR transmitter and receiver [1, 2], BiSAR systems include space-borne BiSAR, air-borne BiSAR, space-borne and air-borne hybrid BiSAR, ground and air-borne/space-borne hybrid BiSAR, and so forth. Nowadays, a novel low-cost and simple-configured BiSAR system is popular which employs an overpass spaceborne SAR as a non-cooperative transmitter and a stationary receiver located on a hill or high building. The noncooperative BiSAR can acquire images with high spatial resolution.

Meanwhile, high spatial resolution could be obtained by using relative motion between antennas and its target [3-7]. However, because of the physical separation between transmitter and receiver, a series of new technological problems still needs to be solved, especially those associated with synchronization [8-10].

One focus on BiSAR imaging is concerned with the synchronization errors, consisting of space synchronization, time synchronization, and phase synchronization. Space synchronization can be solved by the prior knowledge of satellite pass and image opportunity. But the time and phase synchronizations are more complicated and time-consuming.

So far, bistatic image formation algorithms have been basically developed and improved. Rocca firstly introduced Dip Move Out (DMO) method [11] into BiSAR imaging, which is derived from earthquake signal processing methods, converting bistatic echoes to monostatic echoes. Many other researchers were dedicated to approximate methods to obtain two-dimensional spectrum, including the Loffeld Bistatic Formula (LBF) [12], and the series reversion method [13], and Instantaneous Doppler Wavenumber (IDW) [14]. 
Additionally, for the common adaptability, Back Projection (BP) method [15] has been widely used. In the bistatic case with a fixed receiver, only the transmitter contributes to the Doppler history, which makes the different scatterers in the same range cell have different Doppler histories [16,17]. Wong and Yeo put forward a NLCS (Nonlinear Chirp Scaling) algorithm [18], which is only suitable in cases with the small bistatic angle. After that, some extended NLCS and Range Migration Algorithm (RMA) and modified BP algorithm were proposed and gained good results [19-24]. Furthermore, some bistatic system concepts were advanced [25].

However, those synchronization errors could not be eliminated in the absence of accurate orbit data and some important system parameters information, including squint angle, orbit height, satellite velocity, off-nadir angle, and accurate sampling time, while all above imaging algorithms were based on the synchronized echo and thus could not be directly implemented to nonsynchronous echo.

This paper discusses the feasibility of focusing nonsynchronous bistatic echo and introduces a novel imaging algorithm based on the direct signal. This paper is arranged as follows, the basic geometry model and signal model are established in Section 2. Then, the focusing algorithm based on direct signal and image contrast optimization that will be derived in that Section 3. At last, the experimental results will be presented in Sections 4 and 5 will summarize the paper.

\section{Signal Model}

The acquisition geometry of non-cooperative BiSAR systems is shown in Figure 1, where the transmitter is moving along a linear trajectory and the receiver is stationary. The center of scenario is selected as coordinate origin, the $x-y$ plane is tangent to the earth surface and $y$-axis is parallel to the satellite velocity, where the position vector of the transmitter is $\left(x_{t}, y_{t}, z_{t}\right)$ at the azimuth time $\eta=0$, the equivalent satellite velocity is $V$, the incidence angle is $\theta_{t}$, the incidence angle of receiver is $\theta_{r}$, the fixed receiver is located at $\left(x_{r}, y_{r}, z_{r}\right)$, the target position vector is $(x, y, z), R_{t}(\eta ; x, y, z)$ is the range between transmitter and target at azimuth time $\eta, R_{r}$ is the range between target and stationary receiver, $R_{20}$ is the minimum range from the target to the transmitter, and $R_{20 \_ \text {ref }}$ represents the closest range from receiver to the flight path of transmitter. Then, its reflected echo can be expressed as

$$
\begin{aligned}
\operatorname{Echo}(t ; \eta ; x, y, z)= & \operatorname{rect}\left(\frac{t-t_{\text {_delay }}(\eta ; x, y, z)}{T_{p}}\right) \\
& \times \exp \left(j \pi K_{r} \cdot\left(t-t_{\text {_delay }}(\eta ; x, y, z)\right)^{2}\right) \\
& \times \exp \left(-j 2 \pi \frac{R(\eta ; x, y, z)}{c} f_{0}\right),
\end{aligned}
$$

where $t$ represents the fast time, $c$ is the velocity of light, $T_{p}$ is the pulse width, $f_{0}$ is the carrier frequency, $K_{r}$ is the Frequency Modulation (FM) rate, $R(\eta ; x, y, z)$ is the bistatic

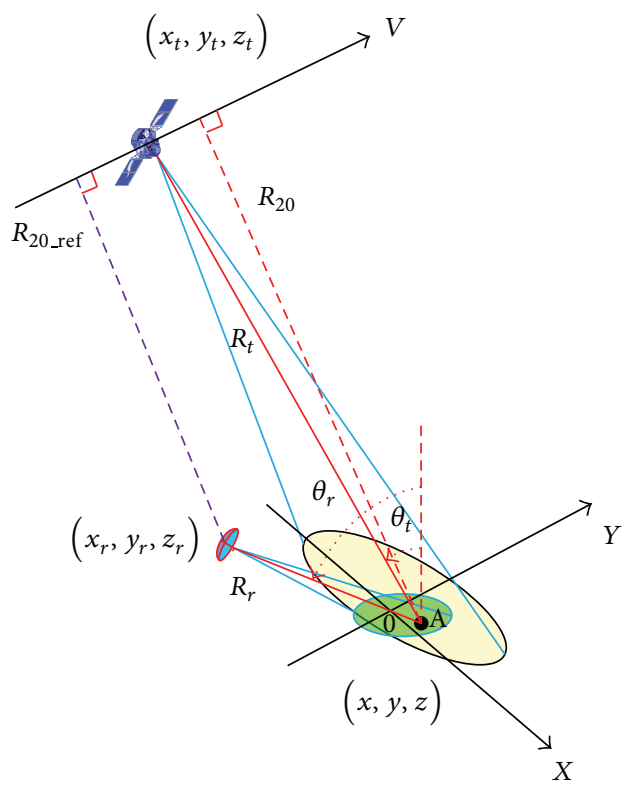

FIGURE 1: Geometry model.

range at azimuth time $\eta$, and $t_{\text {_delay }}(\eta ; x, y, z)$ is the duration time from transmitter to target to receiver at azimuth time $\eta$ :

$$
\begin{gathered}
R(\eta ; x, y, z)=R_{t}(\eta ; x, y, z)+R_{r}, \\
t_{\text {_delay }}(\eta ; x, y, z)=\frac{R(\eta ; x, y, z)}{c}, \\
R_{r}=\sqrt{\left(x-x_{r}\right)^{2}+\left(y-y_{r}\right)^{2}+\left(z-z_{r}\right)^{2}}, \\
R_{t}(\eta ; x, y, z)=\sqrt{\left(x-x_{t}\right)^{2}+\left(y-y_{t}\right)^{2}+\left(z-z_{t}\right)^{2}} .
\end{gathered}
$$

Let us denote the minimum range from the target to the transmitter by $R_{20}$ and the minimum bistatic range sum by $R_{\text {bio }}$; that is,

$$
\begin{gathered}
R_{\text {bio }}=R_{20}+R_{r}, \\
R_{20}=\sqrt{\left(x-x_{t}\right)^{2}+\left(z-z_{t}\right)^{2}} .
\end{gathered}
$$

Transforming the echo signal into range Doppler domain based on the principle of stationary phase (detail in the appendix), we have

$$
\begin{aligned}
\operatorname{Echo}\left(t, f_{\eta}\right)= & W_{r}\left(\frac{K_{e}}{K_{r}} \cdot\left(t-\frac{R_{\mathrm{bio}}+R_{20} \cdot C_{s}\left(f_{\eta}\right)}{c}\right)\right) \\
& \times W_{a}\left(f_{\eta}-f_{\mathrm{dc}}\right) \\
& \times \exp \left(j \pi K_{e} \cdot\left(t-\frac{R_{\mathrm{bio}}+R_{20} \cdot C_{s}\left(f_{\eta}\right)}{c}\right)^{2}\right)
\end{aligned}
$$




$$
\begin{aligned}
& \times \exp \left(-j \frac{2 \pi}{\lambda}\left(R_{\text {bio }}+R_{20} \cdot\left(D\left(f_{\eta}\right)-1\right)\right)\right) \\
& \times \exp \left(-j 2 \pi f_{\eta} \frac{y}{V}\right),
\end{aligned}
$$

where $W_{r}$ and $W_{a}$ are the envelopes in range time domain and azimuth frequency domain, respectively, $f_{\eta}$ is the azimuth frequency, $\lambda$ is the wavelength, $f_{\mathrm{dc}}$ is the Doppler centroid, $K_{e}$ is the FM (Frequency Modulation) rate in range Doppler domain, $K_{\text {src }}$ is the FM rate of SRC filter, $D\left(f_{\eta}\right)$ is the factor of range migration, and $C_{s}\left(f_{\eta}\right)$ is the curvature factor:

$$
\begin{gathered}
K_{e}=\frac{1}{1 / K_{r}-1 / K_{\mathrm{src}}}, \\
K_{\mathrm{src}}=-\frac{V^{2} f_{0}^{2} D^{3}\left(f_{\eta}, V\right)}{\lambda R_{20} f_{\eta}^{2}}, \\
D\left(f_{\eta}\right)=\sqrt{1-\left(\frac{\lambda f_{\eta}}{V}\right)^{2}}, \\
C_{s}\left(f_{\eta}\right)=\frac{1}{D\left(f_{\eta}\right)}-1 .
\end{gathered}
$$

\section{Two-Dimensional Focusing Algorithm}

3.1. Range Focusing Based on Parameters Estimation Using Direct Signal. As for non-cooperative transmitter, passive measurement for transmitted signal is needed [26, 27]. However, only little prior information could be obtained from China Space Agency (CSA), except bandwidth, wavelength, and pulse width, which could be used to estimate the chirprate directly.

Before range processing, the recorded SAR data must be partitioned into two-dimensional by the estimated PRF. The estimated PRF, which is coarse but accurate enough for the proposed algorithm, can be obtained by measuring the positional difference of adjacent peak [28, 29], after range compression is implemented on the one-dimensional direct signal.

Range compression is implemented in frequency domain and then converted the result into time domain. However, as the transmitter is working in the squint mode, the differences between $K_{e}$ and $K_{r}$ could not be ignored. As a result, secondary range compression is inevitable.

In case that the receiver of BiSAR is a stationary receiver, the Doppler centroid and Doppler chirp-rate could be expressed as

$$
\begin{gathered}
f_{\mathrm{dc}}=-\left.\frac{1}{\lambda} \frac{d R(\eta ; x, y, z)}{d \eta}\right|_{\eta=0}=\frac{V \sin \theta}{\lambda}, \\
f_{\mathrm{dr}}=-\left.\frac{1}{\lambda} \frac{d^{2} R(\eta ; x, y, z)}{d \eta^{2}}\right|_{\eta=0}=-\frac{V^{2} \cos ^{3} \theta}{\lambda R_{20}},
\end{gathered}
$$

where $\theta$ is the squint angle of the transmitter.
Substituting (7) and (9) into (6), we have

$$
K_{\mathrm{src}}=-\frac{f_{0}^{2} f_{\mathrm{dr}}}{\cos ^{3} \theta} \frac{\left[1-\left(\left(f_{\eta} / f_{\mathrm{dc}}\right) \sin \theta\right)^{2}\right]^{3 / 2}}{f_{\eta}^{2}}=F\left(f_{\mathrm{dr}}, \theta, f_{\mathrm{dc}}\right) \text {. }
$$

Equation (10) indicates that the FM rate of SRC matched filter is related to Doppler centroid, squint angle, and Doppler chirp-rate. However, these imaging parameters are not feasible for the non-cooperative BiSAR. Fortunately, the direct signal could be used to these key parameters' estimation and the following image focusing.

3.1.1. Estimate the Solution of the Optimal Doppler Chirp-Rate $f_{d r}$. After range compression of direct signal, the Doppler phase could be extracted easily because of its high SNR. The extracted peak phase of direct signal is $\varphi=2 \pi R(\eta ; x, y, z) / \lambda$.

Considering that $\varphi$ is the function of azimuth time $\eta$, thus it can be expanded as Taylor series at $\eta=0$ :

$$
\varphi=\frac{2 \pi}{\lambda}\left(a_{0}+a_{1} \eta+a_{2} \eta^{2}+a_{3} \eta^{3}+a_{4} \eta^{4} \ldots\right)
$$

where $a_{0}, a_{1}, a_{2}, a_{3}, a_{4}, \ldots$ are the coefficients of the expanded phase.

Generally, the first two items in (11) are good enough to guarantee the expansion accuracy, and (11) could be estimated as

$$
\varphi \approx \frac{2 \pi}{\lambda}\left(R_{0}-\sin \theta \cdot V \cdot \eta+\frac{\cos ^{3} \theta}{2 R_{20}}(V \cdot \eta)^{2}\right),
$$

where $R_{0}$ is the bistatic range at $\eta=0$.

According to (9), (12) is rewritten as

$$
\varphi=2 \pi\left(\frac{R_{0}}{\lambda}-\widehat{f}_{\mathrm{dc}} \eta-\frac{1}{2} \widehat{f}_{\mathrm{dr}} \eta^{2}\right)
$$

where $\widehat{f}_{\mathrm{dc}}$ is the estimated Doppler centroid and $\widehat{f}_{\mathrm{dr}}$ is the estimated Doppler chirp-rate.

As can be seen from (13), the peak phase of direct signal is regarded as the quadratic function composed of $\widehat{f}_{\mathrm{dc}}$ and $\widehat{f}_{\mathrm{dr}}$. Obviously, the accurate estimation of $\widehat{f}_{\mathrm{dc}}$ and $\widehat{f}_{\mathrm{dr}}$ could be obtained through quadratic curve fitting between phase $\varphi$ and azimuth time $\eta$.

However, because of the existence of synchronization errors, the estimated Doppler centroid and Doppler chirprate are inaccurate. We denote $\widehat{f}_{\mathrm{dc}}=f_{\mathrm{dc}}+\Delta f_{\mathrm{dc}}$ and $\widehat{f}_{\mathrm{dr}}=$ $f_{\mathrm{dr}}+\Delta f_{\mathrm{dr}}$, where $\Delta f_{\mathrm{dc}}$ and $\Delta f_{\mathrm{dr}}$ are the differences between the estimated value and true value.

It is known to us all that three common problems existed in BiSAR system, including PRF partition errors, introduced phase noise, and different carrier frequency between transmitter and receiver. Of all three errors above, the PRF partition errors and differences of carrier frequency between transmitter and receiver mainly affect the first order term of $\eta$ in (13), while the error caused by phase noise would be guaranteed by system design and stable system capability 
[30-32]. As a result, the impacts introduced by these errors on $f_{\mathrm{dr}}$ can be ignored, and we have $\widehat{f}_{\mathrm{dr}} \approx f_{\mathrm{dr}}$.

After obtaining the accurate estimation of $f_{\mathrm{dr}}$, the FM rate of SRC matched filter thus could be simplified as a quadratic function composed of $f_{\mathrm{dc}}$ and $\theta$. In the following, the simplified two-dimensional optimization will be applied to get optimal $f_{\mathrm{dc}}$ and $\theta$.

3.1.2. The Solution of the Optimal Doppler Centroid $f_{d c}$ and Squint Angle $\theta$. Image contrast refers to the dynamic range of an image, while focusing generally means higher spatial resolution. However, focusing quality could directly be reflected by image contrast [33-35], and the higher the contrast, the better the focused SAR image. The image contrast can be defined as the $=$ standard deviation of the image energy/mean of the image energy.

The optimal $f_{\mathrm{dc}}$ and $\theta$ can be obtained by twodimensional optimization, But the two-dimensional searching is not a good choice. For example, if the loop times in $f_{\mathrm{dc}}$ domain and $\theta$ domain are $M$ and $N$ respectively, obviously, the corresponding $M \times N$, computation capacity is heavy and time-consuming. However, for the low earth orbit, the maximum squint angle of transmitter is around $-4^{\circ}$ even though yaw steering is not applied. Because of introduced synchronization errors, the relationship between estimated Doppler centroid and squint angle could be expressed as $\widehat{f}_{\mathrm{dc}}=\Delta f_{\mathrm{dc}}+(V / \lambda) \sin (\theta) \approx \Delta f+k \cdot \theta$, where $k=V / \lambda$. It indicates that the relationship between $\widehat{f}_{\mathrm{dc}}$ and $\theta$ is approximately linear. As a result, the complicated two-dimensional optimization could be simplified as multi-one-dimensional optimization. Meanwhile, the computation capacity would be reduced to $n \cdot(M+N)$, where $n$ are the times of iteration.

The searching steps are shown as below.

(i) Firstly, a squint angle $\theta$ and a group of $M$ possible Doppler centroids, by which a group of SRC matched filters could be assembled according to (10), should be given. Since the maximum squint angle of transmitter is around $-4^{\circ}$, we set $\theta=-2^{\circ}$ at first in order to decrease the iteration times and improve the searching efficiency.

(ii) Secondly, SRC processing as well as final SAR image could be accomplished based on all assembled matched filters, and we can obtain the image contrast of each picture $C_{k}$, where $k=1: M$. And then, the optimal matched filter could be found based on image contrast optimization, which consists of optimal Doppler centroid $f_{\text {dc_opt }}$ and $\theta$.

(iii) Thirdly, the optimal squint angle $\theta_{\text {_opt }}$ can also be searched by applying the same searching method above based on obtained $f_{\text {dc_opt }}$ and a majority of $N$ given possible squint angles. The image contrast of each picture $D_{n}$ could also be obtained, where $=1: N$.

(iv) Finally, the loop ends when $D_{n}$ and $C_{k}$ satisfy $\left|\left(\max \left(D_{n}\right)-\max \left(C_{k}\right)\right) / \max \left(C_{k}\right)\right|<T_{0}$, where $T_{0}$ is the threshold. Otherwise, let $\theta=\theta_{\text {opt }}$ and repeat the loop. Generally, $T_{0}=10^{-3}$ is selected in the optimization processing [33-35].
TABLE 1: Experimental parameters.

\begin{tabular}{lc}
\hline Wavelength & L band \\
Bandwidth (MHZ) & 60 \\
Sampling rate (MHZ) & 100 \\
PRF (HZ) & 1700 \\
Receiver height $(\mathrm{Km})$ & 24 \\
Scene length $(\mathrm{Km})$ & 1.5 \\
Scene width $(\mathrm{Km})$ & 2 \\
\hline
\end{tabular}

After deriving $\widehat{f}_{\mathrm{dr}}, f_{\mathrm{dc} \_ \text {opt }}$, and $\theta_{\text {_opt }}$, the optimal SRC matched filter $H_{\text {src }}$ could be assembled with the expression shown below:

$$
H_{\text {src }}=\exp \left(-j \pi \frac{f_{t}^{2}}{K_{\text {src }}\left(\widehat{f}_{\mathrm{dr}}, f_{\text {dc_opt } \left._{-}, \theta_{\text {oopt }}\right)}\right)}\right),
$$

where $f_{t}$ is the range frequency.

According to the searching steps above, the nonsynchronous echo of YAOGAN 10 is processed, and the searching results of optimal Doppler centroid $f_{\text {dc_opt }}$ and squint angle $\theta_{\text {-opt }}$ are shown in Figure 2.

Figure 2 shows that the searching results of optimal Doppler centroid and squint angle are $-3811 \mathrm{~Hz}$ and $-2.4^{\circ}$, respectively, with which the optimal SRC matched filter could be constructed.

3.2. RCMC and Focusing in Azimuth Direction. It is known to us all that range in bistatic configuration depends on radar incidence angle and receiver angle $[36,37]$. However, for the lack of the incidence angle of transmitter and receiver and the satellite height based on non-cooperative transmitter, the traditional imaging algorithms could not be applied to Range Cell Migration Correction (RCMC).

However, considering the peculiarity of low receiver, narrow beam, and small scene in our real BiSAR system (parameters are shown in Table 1), the spatial variation of RCM could be ignored, and we can realize the Range Cell Migration Correction (RCMC) by compensating the RCM of echo with that of direct signal.

As can be seen from (4), the range history of a random target in range Doppler domain is expressed as

$$
R=\frac{R_{20}}{D\left(f_{\eta}\right)}+R_{r}
$$

According to (15), we denote the RCM of a random target as

$$
\mathrm{RCM}=\frac{R_{20}}{D\left(f_{\eta}\right)}-R_{20}
$$

It could be seen from (16) that RCM varies with range time and azimuth frequency, and the difference of RCM between a random target and stationary receiver is

$$
\Delta \mathrm{RCM}=\frac{R_{20}-R_{20 \_ \text {ref }}}{D\left(f_{\eta}\right)}-\left(R_{20}-R_{20 \_ \text {ref }}\right) .
$$




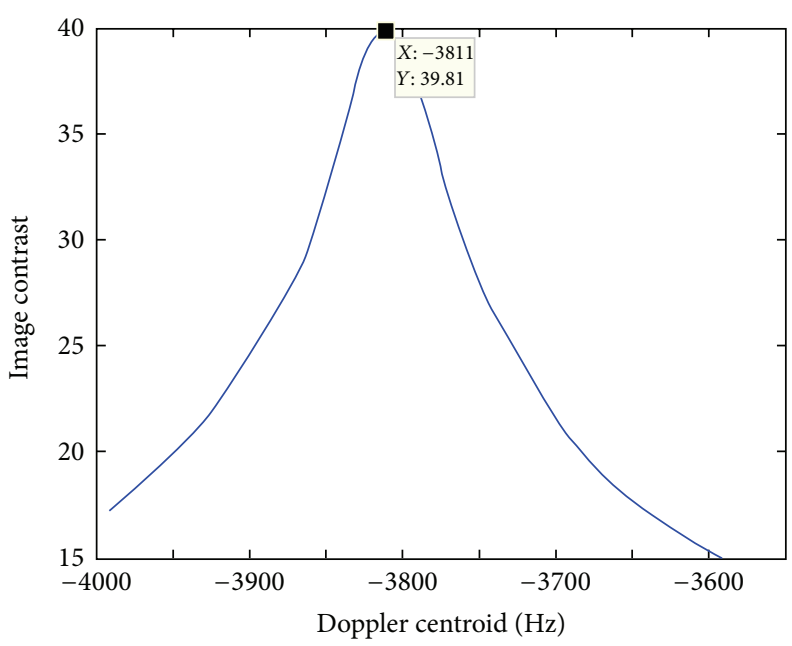

(a) Doppler centroid

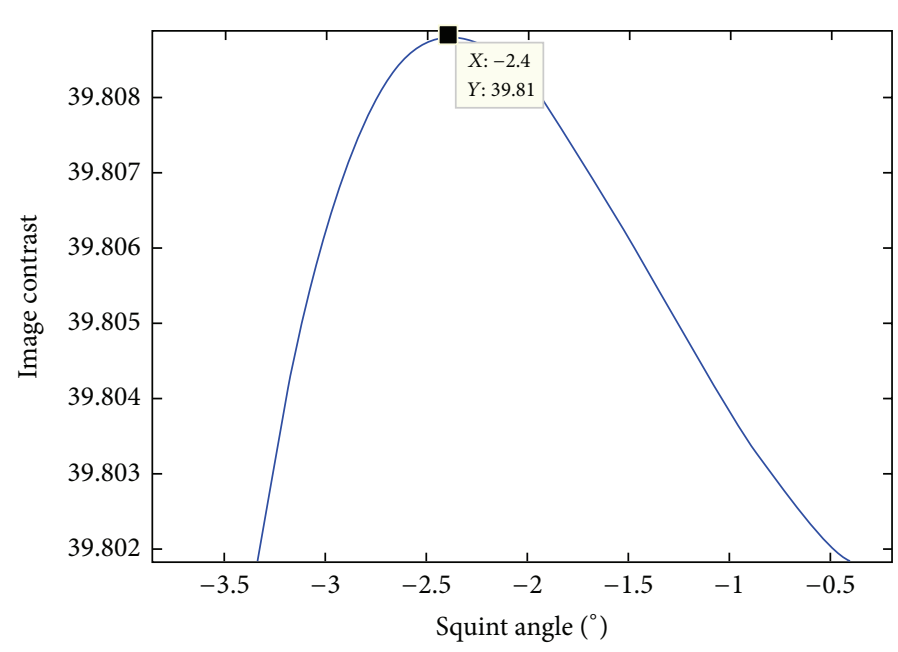

(b) Squint angle

FIGURE 2: Searching results of twice one-dimensional optimization.

Obviously, the spatial variation of RCM cannot be ignored, until (17) is smaller than half of a range cell.

In order to extract accurate RCM of direct signal, curve fitting is needed. In normal cases, quadratic curve fitting is enough to satisfy the precision. (The processing result of real data is shown in Figure 5).

For focus in azimuth direction, the echo in range Doppler domain should be multiplied by the conjugate of the peak phase of direct signal in each range gate, while it is easy to extract the peak phase in range Doppler domain for the high energy of direct signal.

The expression of azimuth matched filter $H_{a}$ could be written as

$$
H_{a}=\exp \left(j \frac{2 \pi}{\lambda} R_{20 \_ \text {ref }} D\left(f_{\eta}\right)\right) .
$$

According to (4) and (18), the difference of the peak phase between a random target and direct signal in range Doppler domain is expressed as

$$
\Delta \varphi=\frac{2 \pi}{\lambda}\left[\left(R_{20}-R_{20 \_ \text {ref }}\right) D\left(f_{\eta}\right)+R_{r}\right] .
$$

In order to focus on the whole scene, the maximum quadratic phase error of echo should satisfy $|\Delta \varphi|_{\max }<\pi / 4$. By all appearances, from (17) and (19), the size of imaging scene is limited. Using the parameters in Table 1, the largest scene that can be focused is $2 \mathrm{~km} \times 2 \mathrm{~km}$, and it meets our demands.

After the azimuth compression, the slant range image in two-dimensional time domain can be obtained with an azimuth IFFT.

The flow chart of the proposed algorithm is shown in Figure 3.

It could be seen from Figure 3(a) that the proposed algorithm is mainly composed of four parts. Including, range compression, optimal SRC, Range Cell Migration Correction (RCMC), and azimuth compression. The core part shown in Figure 3(b), which consists of multi-one-dimensional optimization, can search the optimal $f_{\mathrm{dc}}$ and $\theta$ accurately and efficiently. As for each optimization, searching intervals, including $\Delta f$ and $\Delta \theta$, should be defined based on precision requirements.

\section{BiSAR Experiment}

To testify the validity of the proposed algorithm, BiSAR experiments based on the opportunity illuminator are carried out in JiangSu province of China, and the nonsynchronous echo of China YAOGAN 10 is used.

The BiSAR experimental hardware adopted in the bistatic experiments is shown in Figure 4, where different components have been labeled, while the stationary receiver is located on the building with the height of $109 \mathrm{~m}$.

There are two kinds of channels in the receiver, which are radar channel to collect the reflected echo from target area and reference channel to collect directly illuminated signal from the transmitter for synchronization processing.

At the bottom of Figure 4, an optic picture of the target area is given. It is a very good target area because different targets including roads, river, and factory rooms, were included and could be used to test the different bistatic responses from the target area. The bistatic experiments were carried out in 2012.

When the optimal Doppler centroid and squint angle are obtained based on image contrast optimization (shown in Figure 2), the optimal SRC matched filter can be applied to perform the SRC perfectly. After that, the RCM of the direct signal could be extracted to compensate the echo in two-dimensional frequency domain. The results after SRC and RCMC are shown, respectively, in Figure 5, while the extracted RCM before and after curve fitting is shown in Figure 6.

After RCMC is finished, the peak phase of the direct signal can be extracted in range Doppler domain and used to match the echo in azimuth. 

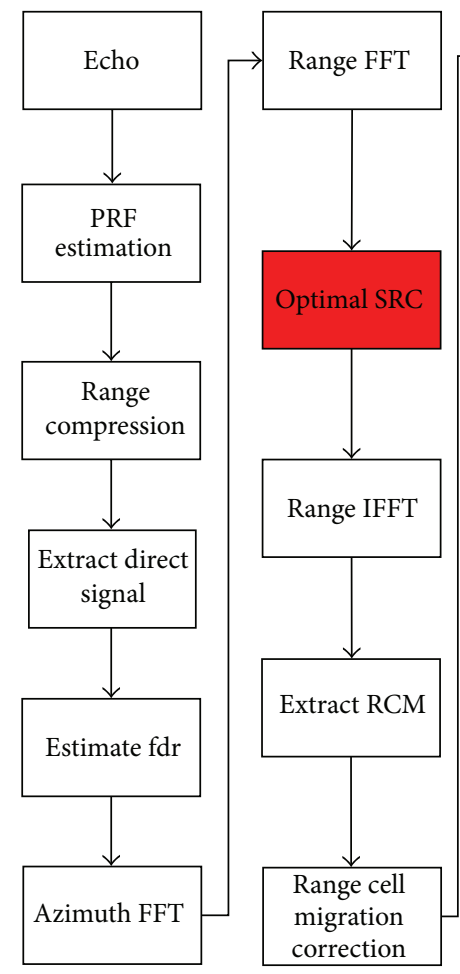

(a) Main part

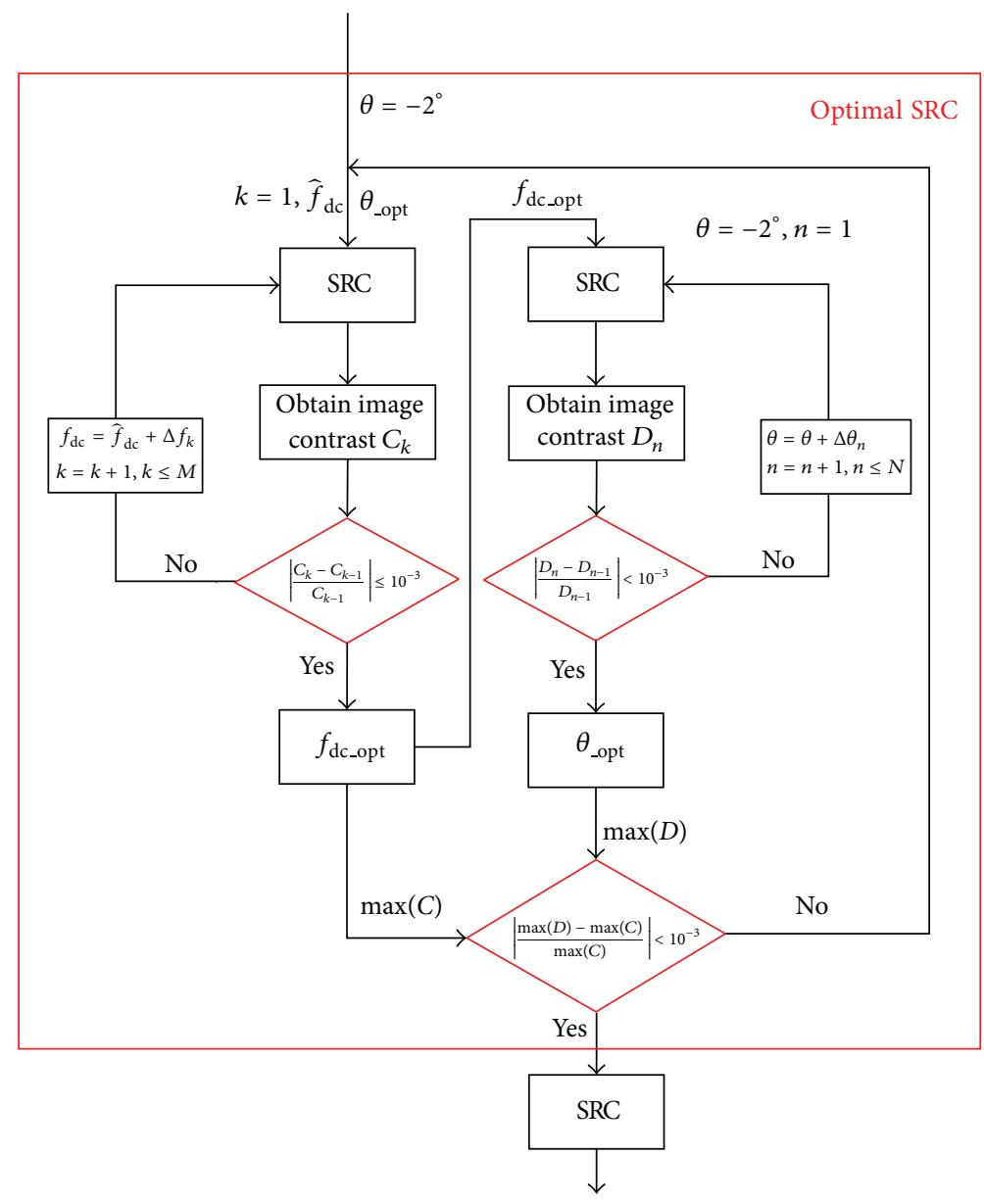

(b) Core part

Figure 3: The flow chart of the proposed algorithm.

For a better understanding, a classic NLCS algorithm [22] is also used to process the nonsynchronous echo of YAOGAN 10 and the comparison is shown in Figure 7.

It can be seen from Figure 7 that (a) is obviously better focused than (b), and the river, roads, rooms, and so forth can be clearly seen in the red circles. It is suggested from the above results that the proposed algorithm has the ability to process nonsynchronous echo.

The slant range image after geometry correction and the Google optic image are also shown, respectively, in Figure 8, where the coincidence between the bistatic and optical images is found.

It is suggested from Figure 8(b) that the places we are interested in are not influenced, the main targets, such as factory rooms, roads, and river in the bistatic image have a very good accordance with the optical one.

\section{Conclusion}

Based on searching of the optimal SRC matched filter from the information of direct signal, this paper presented a novel imaging method for the nosynchronous echo based on the special mode of BiSAR with a stationary receiver. Then, The RCM and peak of direct signal were extracted for the azimuth focusing. The experiment data with china's YAOGAN 10 SAR as the transmitter are processed by the proposed method. The images are also geometrically corrected and then visually compared with a Google image of the corresponding region. The good accordance shows the imaging algorithm's effectiveness and the fascinating potential application of this non-cooperative BiSAR system in the future.

\section{Appendix}

Based on (1), the expression after range FFT could be expressed as

$$
S\left(f_{t}, \eta\right)=\int_{-\infty}^{+\infty} \operatorname{Echo}(t ; \eta ; x, y, z) \cdot \exp \left(-j 2 \pi f_{t} t\right) \cdot d t
$$

where $f_{t}$ is the range frequency. 


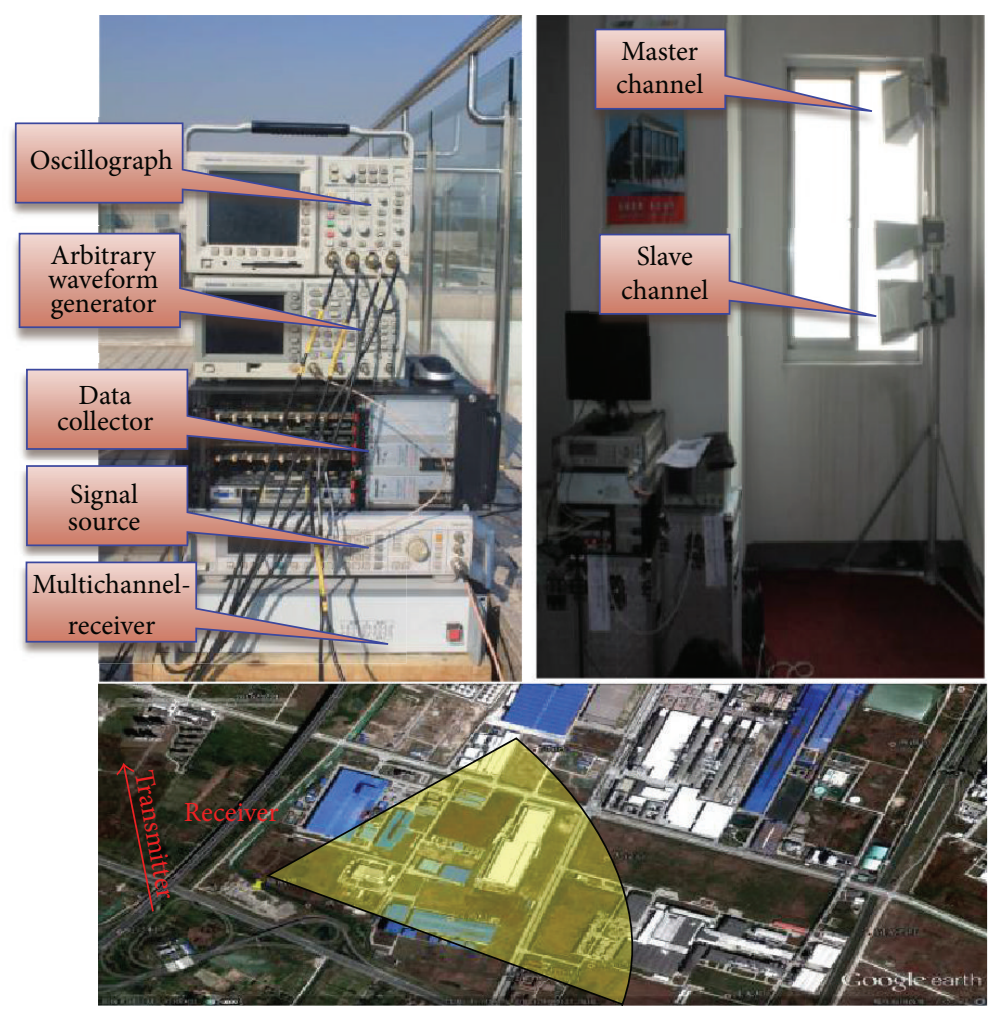

FIGURE 4: Bistatic SAR system.

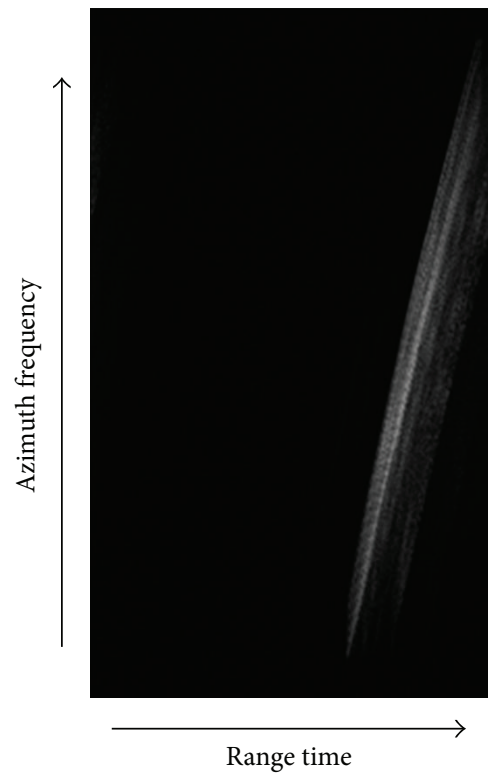

(a) After SRC

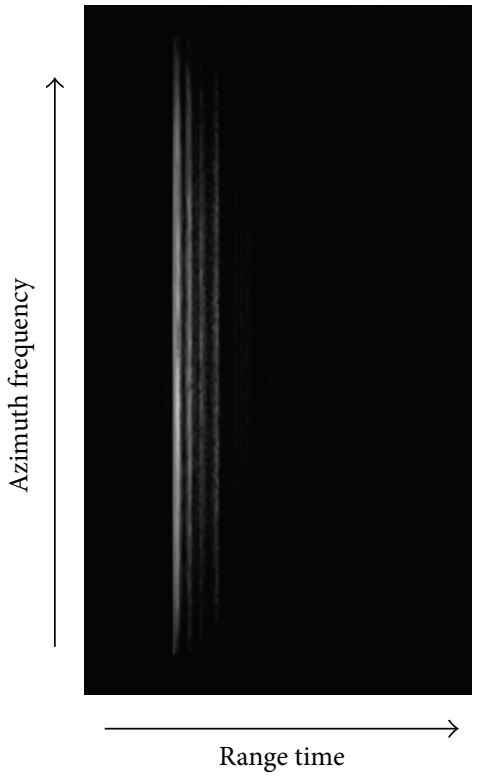

(b) After RCMC

FIgURE 5: The results after SRC and RCMC.

The phase in the integral is

$$
\begin{aligned}
\theta(t)= & \pi K_{r} \cdot\left(t-\frac{R(\eta ; x, y, z)}{c}\right)^{2} \\
& -2 \pi \frac{R(\eta ; x, y, z)}{c} f_{0}-2 \pi f_{t} t .
\end{aligned}
$$

According to the principle of stationary phase, the stationary phase point is

$$
t=\frac{f_{t}}{K_{r}}+\frac{R(\eta ; x, y, z)}{c} .
$$




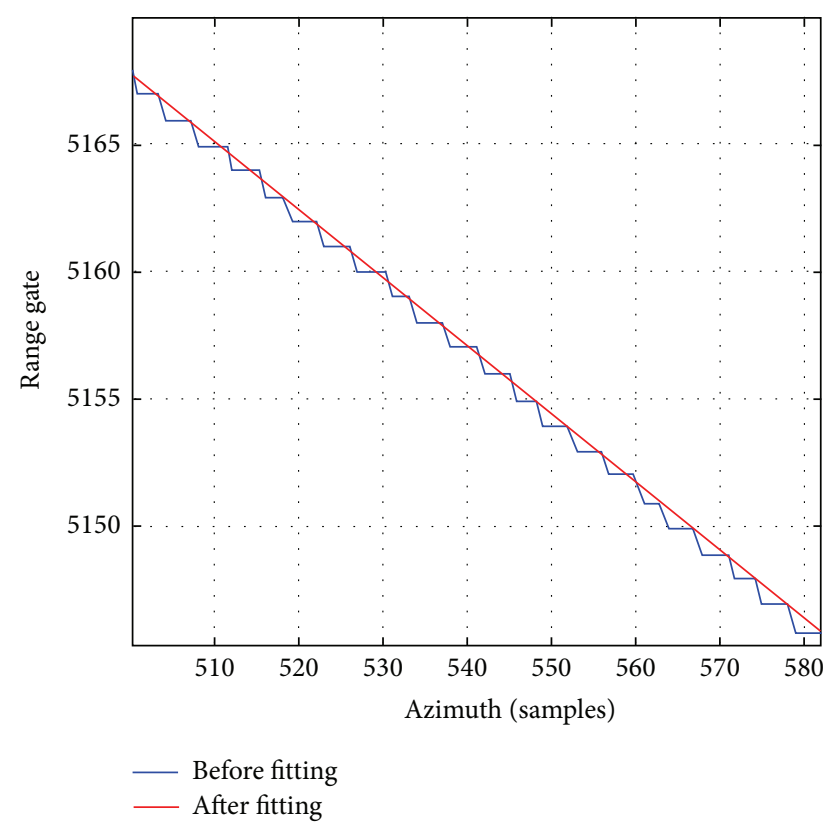

FIGURE 6: The extracted RCM before and after curve fitting.

Substituting (A.3) into (A.1), and we get that

$$
\begin{aligned}
S\left(f_{t}, \eta\right)= & w_{r}\left(f_{t}\right) w_{a}(\eta) \exp \left(-j \pi \frac{f_{t}^{2}}{K_{r}}\right) \\
& \times \exp \left(-j 2 \pi \frac{\left(f_{0}+f_{t}\right) R(\eta ; x, y, z)}{c}\right) .
\end{aligned}
$$

After an azimuth FFT operation, the expression of (A.4) could be written as

$$
S\left(f_{t}, f_{\eta}\right)=\int_{-\infty}^{+\infty} S\left(f_{t}, \eta\right) \cdot \exp \left(-j 2 \pi f_{\eta} \eta\right) \cdot d \eta
$$

Obviously, the phase in the integral is

$$
\theta_{\eta}=-\pi \frac{f_{t}^{2}}{K_{r}}-2 \pi \frac{\left(f_{0}+f_{t}\right) R(\eta ; x, y, z)}{c}-2 \pi f_{\eta} \eta .
$$

In the meantime, (A.6) could be rewritten as

$$
\begin{aligned}
\theta_{\eta}= & -\pi \frac{f_{t}^{2}}{K_{r}}-2 \pi \frac{\left(f_{0}+f_{t}\right) \cdot R(\eta ; x, y, z)}{c} \\
& -2 \pi f_{\eta} \eta-2 \pi \frac{\left(f_{0}+f_{t}\right) \cdot R(\eta ; x, y, z)}{c} .
\end{aligned}
$$

Based on the principle of stationary phase, the stationary phase point of (A.7) is

$$
\eta=-\frac{c R_{20} f_{\eta}}{\left(f_{0}+f_{t}\right) V^{2} \sqrt{1-\left(c^{2} f_{\eta}^{2} / V^{2}\left(f_{0}+f_{t}\right)^{2}\right)}}-\frac{y}{V} .
$$

Substituting (A.8) into (A.7), the expression of echo in 2D frequency domain could be shown as

$$
\begin{aligned}
S\left(f_{t}, f_{\eta}\right)= & w_{r}\left(f_{t}\right) w_{a}\left(f_{\eta}-f_{\mathrm{dc}}\right) \\
& \times \exp \left(-j 2 \pi \frac{R_{20}}{c} f_{0} \sqrt{1-\frac{c^{2} f_{\eta}^{2}}{v^{2} f_{0}^{2}}+2 \frac{f_{t}}{f_{0}}+\frac{f_{t}^{2}}{f_{0}^{2}}}\right) \\
& \times \exp \left(-j \pi \frac{f_{t}^{2}}{K_{r}}\right) \exp \left(-j 2 \pi \frac{\left(f_{0}+f_{t}\right)}{c} R_{r}\right) \\
& \times \exp \left(-j 2 \pi f_{\eta} \frac{y}{V}\right) .
\end{aligned}
$$

In order to get the expression of echo in range Doppler domain, we have

$$
S\left(t, f_{\eta}\right)=\int_{-\infty}^{+\infty} S\left(f_{t}, f_{\eta}\right) \cdot \exp \left(j 2 \pi f_{t} \eta\right) \cdot d f_{\eta} .
$$

Let us denote $D\left(f_{\eta}\right)=\sqrt{1-\left(c^{2} f_{\eta}^{2} / v^{2} f_{0}^{2}\right)}$ and expand $\sqrt{1-\left(c^{2} f_{\eta}^{2} / v^{2} f_{0}^{2}\right)+2\left(f_{t} / f_{0}\right)+\left(f_{t}^{2} / f_{0}^{2}\right)}$ as the polynomial function of $f_{t}$ by Taylor expansion; that is,

$$
\begin{aligned}
\sqrt{1-\frac{c^{2} f_{\eta}^{2}}{v^{2} f_{0}^{2}}+2 \frac{f_{t}}{f_{0}}+\frac{f_{t}^{2}}{f_{0}^{2}} \approx} & 1-\frac{c^{2} f_{\eta}^{2}}{v^{2} f_{0}^{2}} \\
& +\frac{f_{t}}{f_{0}\left(1-\left(c^{2} f_{\eta}^{2} / v^{2} f_{0}^{2}\right)\right)} \\
& -\frac{f_{t}^{2} c^{2} f_{\eta}^{2}}{8 V^{2} f_{0}^{4}\left(1-\left(c^{2} f_{\eta}^{2} / v^{2} f_{0}^{2}\right)\right)^{3}} .
\end{aligned}
$$

Consequently, the phase of (A.9) could be simplified as

$$
\begin{aligned}
\theta\left(f_{\eta}\right)= & -\frac{2 \pi R_{20}}{c}\left[D\left(f_{\eta}\right)+\frac{f_{t}}{f_{0} D\left(f_{\eta}\right)}-\frac{f_{t}^{2} c^{2} f_{\eta}^{2}}{4 V^{2} f_{0}^{4} D^{3}\left(f_{\eta}\right)}\right] \\
& -\frac{f_{t}^{2}}{K_{r}}-2 \pi \frac{\left(f_{0}+f_{t}\right)}{c} R_{r}-2 \pi f_{\eta} \frac{y}{V}+2 \pi f_{t} t .
\end{aligned}
$$
(A.12)

Similarly, we could get the stationary phase point of

$$
f_{t}=\frac{K_{r}}{1-K_{r} \cdot Z}\left[t-\left(\frac{R_{r}}{c}+\frac{R_{20}}{c \cdot D\left(f_{\eta}\right)}\right)\right],
$$

where

$$
Z=-\frac{\lambda R_{20} f_{\eta}^{2}}{V^{2} f_{0}^{2} D^{3}\left(f_{\eta}, V\right)}
$$




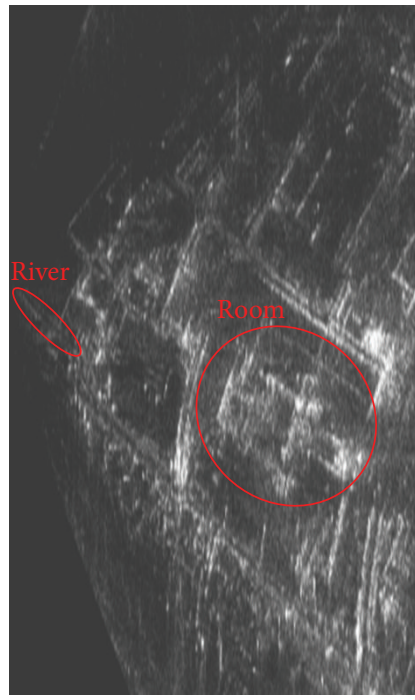

(a) The result of the proposed algorithm

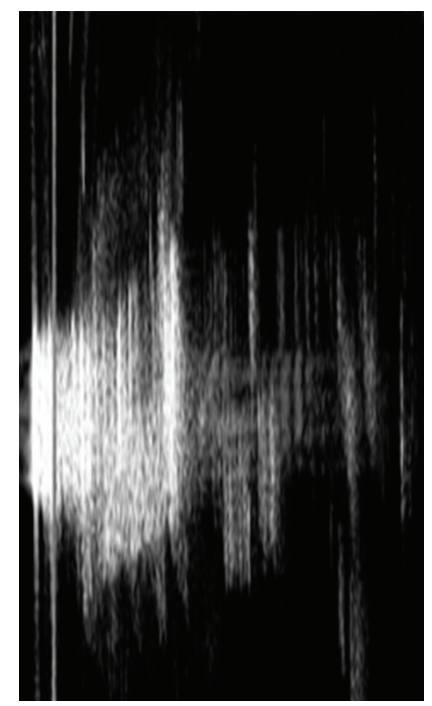

(b) The result of the classical NLCS algorithm

FIGURE 7: Bistatic images from different algorithms.

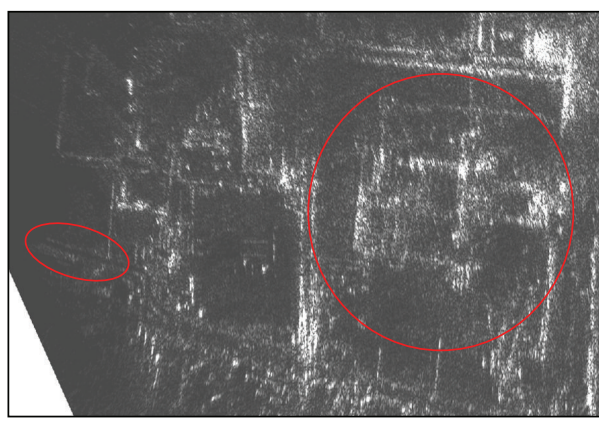

(a) Image after geometry correction

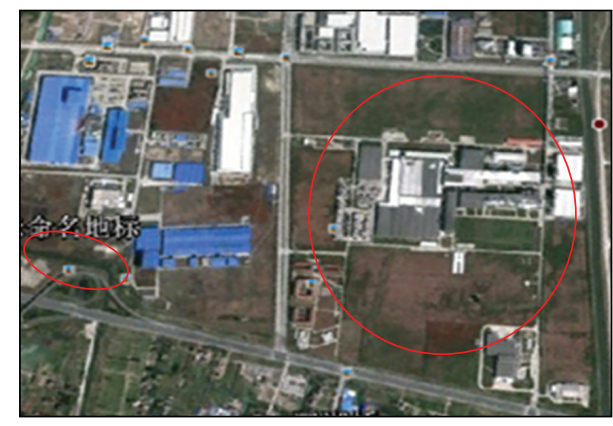

(b) Google image

FIgURE 8: Bistatic SAR and optical images.

The expression of echo in range Doppler domain could be obtained by combining (A.9), (A.10), (A.13); and (A.14):

$$
\begin{aligned}
\operatorname{Echo}\left(t, f_{\eta}\right)= & W_{r}\left(\frac{K_{e}}{K_{r}} \cdot\left(t-\frac{R_{\text {bio }}+R_{20} \cdot C_{s}\left(f_{\eta}\right)}{c}\right)\right) \\
& \times W_{a}\left(f_{\eta}-f_{\text {dc }}\right) \\
& \times \exp \left(j \pi K_{e} \cdot\left(t-\frac{R_{\text {bio }}+R_{20} \cdot C_{s}\left(f_{\eta}\right)}{c}\right)^{2}\right) \\
& \times \exp \left(-j \frac{2 \pi}{\lambda}\left(R_{\text {bio }}+R_{20} \cdot\left(D\left(f_{\eta}\right)-1\right)\right)\right) \\
& \times \exp \left(-j 2 \pi f_{\eta} \frac{y}{V}\right) .
\end{aligned}
$$

\section{Conflict of Interests}

The authors declare that there is no conflict of interests regarding the publication of this paper.

\section{Acknowledgment}

This work was supported by the key project of National Natural Science Foundation of China (Grant nos. 61225005, 61032009, 61172177, 61120106004, and 60890073).

\section{References}

[1] A. Moccia, G. Salzillo, M. D’Errico, G. Rufino, and G. Alberti, "Performance of spaceborne bistatic synthetic aperture radar," IEEE Transactions on Aerospace and Electronic Systems, vol. 41, no. 4, pp. 1383-1395, 2005.

[2] M. Rodriguez-Cassola, S. V. Baumgartner, G. Krieger, and A. Moreira, "Bistatic TerraSAR-X/F-SAR spaceborne-airborne SAR experiment: description, data processing, and results," 
IEEE Transactions on Geoscience and Remote Sensing, vol. 48, no. 2, pp. 781-794, 2010.

[3] S. H. Lim, J. H. Han, S. Y. Kim, and N. H. Myung, "Azimuth beam pattern synthesis for air-borne sar system optimization," Progress in Electromagnetics Research, vol. 106, pp. 295-309, 2010.

[4] S.-J. Wei, X.-L. Zhang, J. Shi, and G. Xiang, "Sparse reconstruction for SAR imaging based on compressed sensing," Progress in Electromagnetics Research, vol. 109, pp. 63-81, 2010.

[5] W. Xu, P. P. Huang, and Y. K. Deng, "Multi-channel SPCMBtops sar for high-resolution wide-swath imaging," Progress in Electromagnetics Research, vol. 116, pp. 533-551, 2011.

[6] S.-J. Wei, X.-L. Zhang, and J. Shi, "Linear array SAR imaging via compressed sensing," Progress in Electromagnetics Research, vol. 117, pp. 299-319, 2011.

[7] Q. Liu, W. Hong, W.-X. Tan, Y. Lin, Y.-P. Wang, and Y.-R. $\mathrm{Wu}$, "An improved polar format algorithm with performance analysis for geosynchronous circular SAR 2D imaging," Progress in Electromagnetics Research, vol. 119, pp. 155-170, 2011.

[8] M. Younis, R. Metzig, and G. Krieger, "Performance prediction of a phase synchronization link for bistatic SAR," IEEE Geoscience and Remote Sensing Letters, vol. 3, no. 3, pp. 429-433, 2006.

[9] M. Younis, R. Metzig, G. Krieger, M. Bacmnann, and R. Klein, "Performance prediction and verification for the synchronization link of TanDEM-X," in Proceedings of the IEEE International Geoscience and Remote Sensing Symposium (IGARSS '07), pp. 5206-5209, June 2007.

[10] M. Weiß, "Synchronisation of bistatic radar systems," in Proceedings of the IEEE International Geoscience and Remote Sensing Symposium (IGARSS '04), pp. 1750-1753, September 2004.

[11] D. D’Aria, A. M. Guarnieri, and F. Rocca, "Focusing bistatic synthetic aperture radar using dip move out," IEEE Transactions on Geoscience and Remote Sensing, vol. 42, no. 7, pp. 1362-1376, 2004.

[12] O. Loffeld, H. Nies, V. Peters, and S. Knedlik, "Models and useful realtions for bistatic SAR processing," IEEE Transactions on Geoscience and Remote Sensing, vol. 42, no. 10, pp. 2031-2038, 2004.

[13] Y. L. Neo, F. Wong, and I. G. Cumming, "A two-dimensional spectrum for bistatic SAR processing using series reversion," IEEE Geoscience and Remote Sensing Letters, vol. 4, no. 1, pp. 93-96, 2007.

[14] Z. Zhenhua, X. Mengdao, D. Jinshan, and B. Zheng, "Focusing parallel bistatic SAR data using the analytic transfer function in the wavenumber domain," IEEE Transactions on Geoscience and Remote Sensing, vol. 45, no. 11, pp. 3633-3645, 2007.

[15] Y. Ding and D. C. Munson Jr., "A fast back-projection algorithm for bistatic SAR imaging," in Proceedings of the International Conference on Image Processing (ICIP'02), pp. 449-452, September 2002.

[16] C. Hu, T. Zeng, and H.-B. Zeng, "Bistatic synthetic aperture radar point spread function characteristic analysis," Journal of Beijing Institute of Technology, vol. 16, no. 2, pp. 193-196, 2007.

[17] T. Zeng, M. Cherniakov, and T. Long, "Generalized approach to resolution analysis in BSAR," IEEE Transactions on Aerospace and Electronic Systems, vol. 41, no. 2, pp. 461-474, 2005.

[18] F. H. Wong and T. S. Yeo, "New applications of nonlinear chirp scaling in SAR data processing," IEEE Transactions on Geoscience and Remote Sensing, vol. 39, no. 5, pp. 946-953, 2001.
[19] T. Zeng, F. Liu, C. Hu, and T. Long, "Image formation algorithm for asymmetric bistatic SAR systems with a fixed receiver," IEEE Transactions on Geoscience and Remote Sensing, vol. 50, no. 11, pp. 4684-4698, 2012.

[20] J. J. Wu, J. Y. Yang, Y. L. Huang, Z. Liu, and H. G. Yang, "A new look at the point target reference spectrum for bistatic SAR," Progress in Electromagnetics Research, vol. 119, pp. 363-379, 2011.

[21] D. M. Guo, H. P. Xu, and J. W. Li, "Extended wavenumber domain algorithm for highly squinted sliding spotlight sar data processing," Progress in Electromagnetics Research, vol. 114, pp. 17-32, 2011.

[22] X. Qiu, D. Hu, and C. Ding, "An improved NLCS algorithm with capability analysis for one-stationary BiSAR," IEEE Transactions on Geoscience and Remote Sensing, vol. 46, no. 10, pp. 3179-3186, 2008.

[23] R. Wang, F. Li, and T. Zeng, "Bistatic SAR experiment, processing and results in spaceborne/stationary configuration," in Proceedings of the IEEE CIE 6th International Conference on Radar (RADAR '11), pp. 393-397, October 2011.

[24] S. Reuter, F. Behner, H. Nies, O. Loffeld, D. Matthes, and J. Schiller, "Development and experiments of a passive SAR receiver system in a bistatic spaceborne/stationary configuration," in Proceedings of the 30th IEEE International Geoscience and Remote Sensing Symposium (IGARSS '10), pp. 118-121, Honolulu, Hawaii, USA, July 2010.

[25] G. Krieger, H. Fiedler, D. Hounam, and A. Moreira, "Analysis of system concepts for bi- and multi-static SAR missions," in Proceedings of theIEEE International Geoscience and Remote Sensing Symposium (IGARSS '03), vol. 2, pp. 770-772, July 2003.

[26] V. Kubica and X. Neyt, "Passive SAR imaging using the ASAR instrument of ENVISAT as transmitter of opportunity," in Proceedings of the 9th European Conference on Synthetic Aperture Radar (EUSAR '12), pp. 275-278, Nuremberg, Germany, April 2012.

[27] P. Samczynski, K. Kulpa, M. Malanowski, P. Krysik, and L. Maslikowski, "Trial results on passive SAR measurement using the Envisat-1 satellite as an illuminator of opportunity," in Proceedings of the 9th European Conference on Synthetic Aperture Radar (EUSAR '12), pp. 291-294, Nuremberg, Germany, April 2012.

[28] P. López-Dekker, J. J. Mallorquí, P. Serra-Morales, and J. SanzMarcos, "Phase synchronization and doppler centroid estimation in fixed receiver bistatic SAR systems," IEEE Transactions on Geoscience and Remote Sensing, vol. 46, no. 11, pp. 3459-3471, 2008.

[29] P. López-Dekker, J. J. Mallorqui, P. Serra-Morales, and J. SanzMarcos, "Phase and temporal synchronization in bistatic SAR systems using sources of opportunity," in Proceedings of the IEEE International Geoscience and Remote Sensing Symposium (IGARSS '07), pp. 97-100, June 2007.

[30] W. Tian, S. Hu, and T. Zeng, "A frequency synchronization scheme based on PLL for BiSAR and experiment result," in Proceedings of the 9th International Conference on Signal Processing (ICSP '08), pp. 2425-2428, October 2008.

[31] W. Tian, H. Liu, and T. Zeng, "Frequency and time synchronization error analysis based on generalized signal model for bistatic SAR," in Proceedings of the IET International Radar Conference 2009, April 2009.

[32] W. Tian, T. Long, J. Yang, and X. Yang, "Combined analysis of time\&frequency synchronization error for BiSAR," in Proceedings of the 6th International Conference on Radar (RADAR '11), pp. 388-392, October 2011. 
[33] F. Berizzi, G. Corsini, M. Diani, and M. Veltroni, "Autofocus of wide azimuth angle SAR images by contrast optimisation," in Proceedings of the International Geoscience and Remote Sensing Symposium (IGARSS '96), pp. 1230-1232, May 1996.

[34] D. E. Wahl, P. H. Eichel, D. C. Ghiglia, and C. V. Jakowatz Jr., "Phase gradient autofocus-a robust tool for high resolution SAR phase correction," IEEE Transactions on Aerospace and Electronic Systems, vol. 30, no. 3, pp. 827-835, 1994.

[35] K. Zhang, "COPA - a fast and universal phase adjustment for SAR," Journal of Northwestern Polytechnical University, vol. 26, no. 4, pp. 481-487, 2008.

[36] F. Behner and S. Reuter, "HITCHHIKER-hybrid bistatic high resolution SAR," in Proceedings of the 8th European Conference on Experiment using a Stationary Receiver and TerraSAR-X Transmitter, Synthetic Aperture Radar (EUSAR '10), pp. 1-4, Aachen, Germany, June 2010.

[37] P. Samczynski and K. Kulpa, "Passive SAR imaging using a satellite pulsed radar as an illuminator of opportunity," in Proceedings of the 13th International Radar Symposium (IRS '12), pp. 157-161, 2012. 


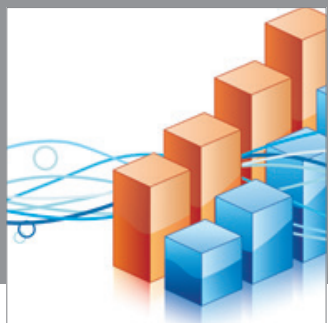

Advances in

Operations Research

mansans

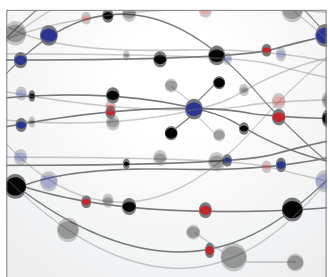

The Scientific World Journal
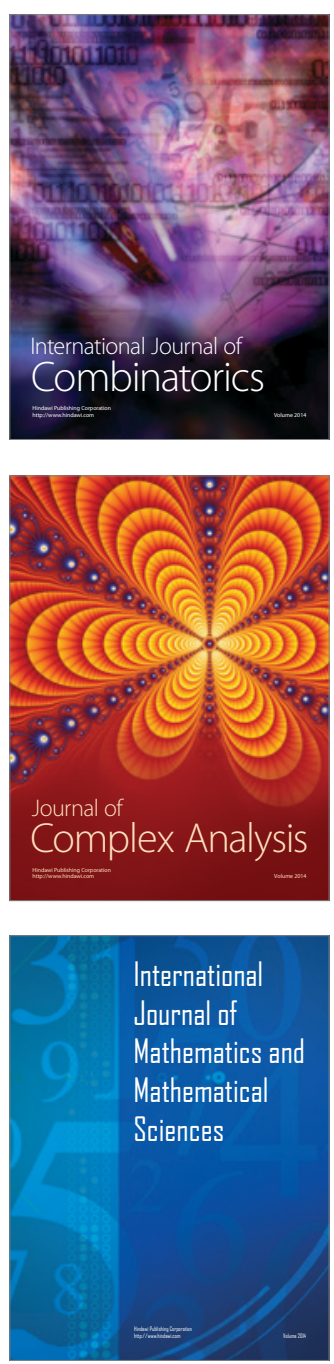
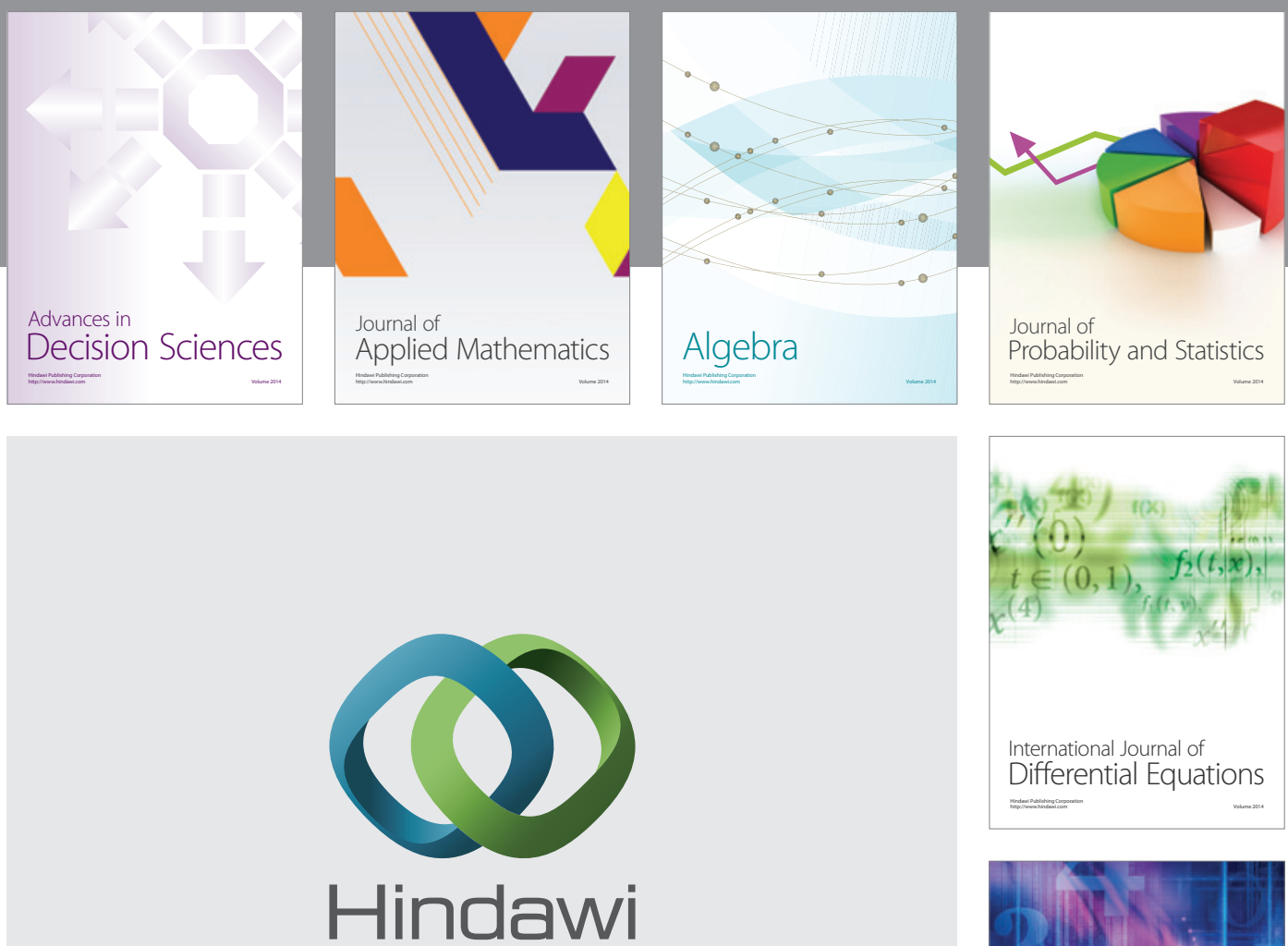

Submit your manuscripts at http://www.hindawi.com
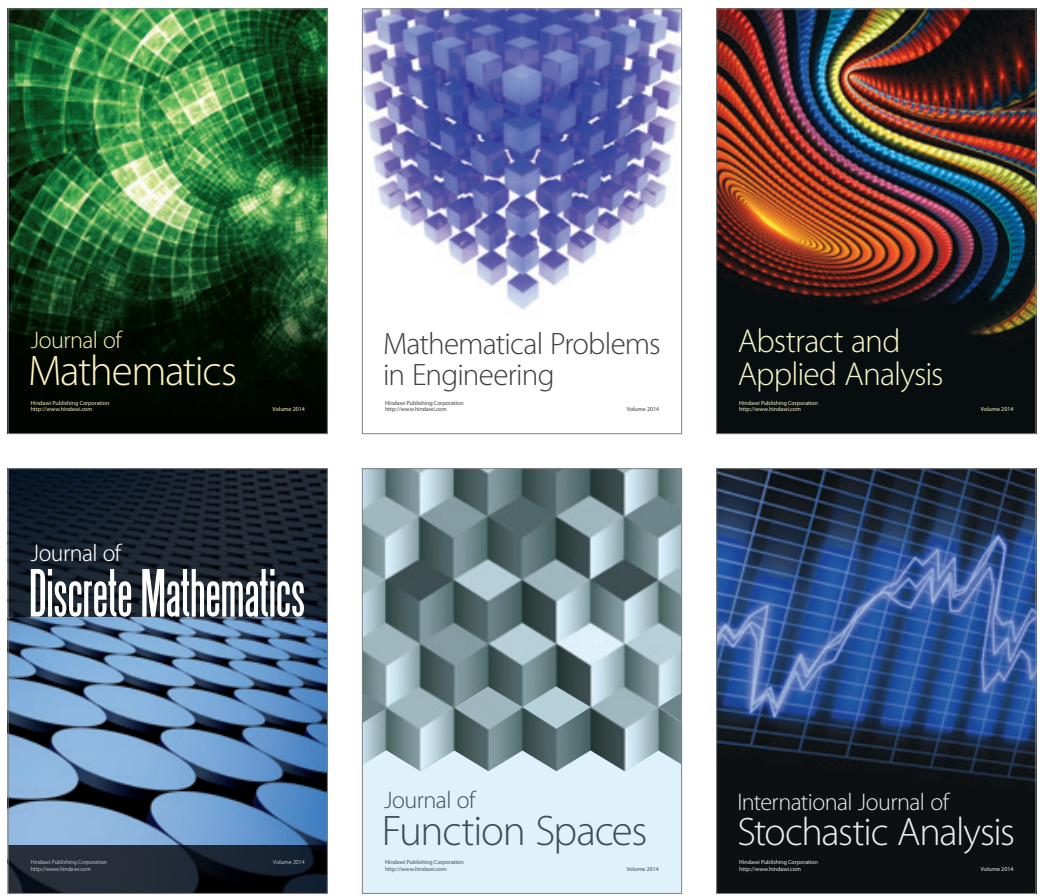

Journal of

Function Spaces

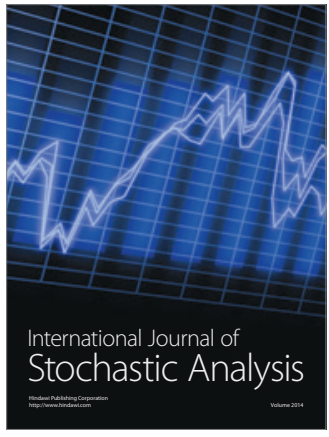

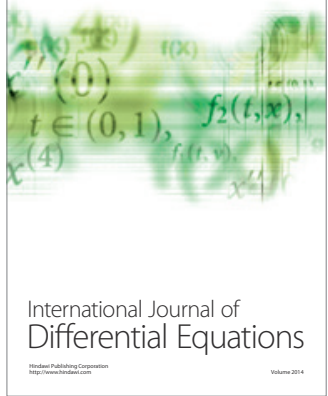
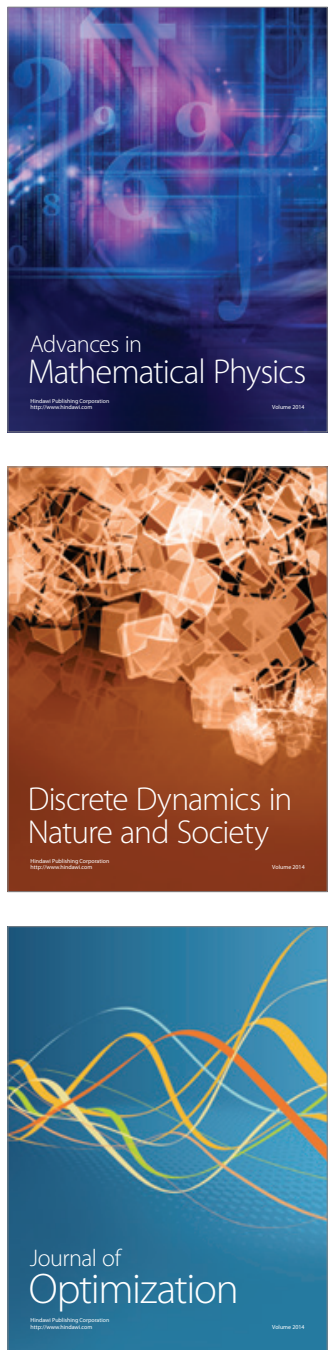